\title{
EL ORDEN DE PALABRAS EN LOS ESTUDIOS GRAMATICALES: UN RECORRIDO A TRAVÉS DE DIFERENTES PERSPECTIVAS LINGÜÍSTICAS
}

\section{WORD ORDER IN GRAMMAR STUDIES: AN OVERVIEW OF DIFFERENT LINGUISTIC PERSPECTIVES ${ }^{1}$}

\author{
A la memoria de la Dra. Anamaría Harvey \\ Enrique Sologuren Insúa \\ Universidad de Los Andes, Chile \\ esologuren@miuandes.cl \\ María Natalia Castillo Fadić \\ Pontificia Universidad Católica de Chile, Chile \\ mcastilf@uc.cl
}

\section{Resumen}

El presente artículo analiza el fenómeno gramatical del orden de palabras. Para esto, propone un recorrido por diferentes aproximaciones y enfoques teóricos que han intentado sistematizar y explicar las alteraciones que los hablantes o escritores realizan al orden sintáctico regular con diferentes propósitos, conscientes o no de las posibilidades que les brinda la gramática de su lengua materna. El análisis se centra en tres aspectos fundamentales: a) la teorización sobre el lenguaje, b) la conceptualización del objeto en diferentes corrientes y c) su estudio en el ámbito de la lengua española. La revisión propuesta permite comprender el problema de manera integral al interior de los estudios gramaticales y sentar las bases para investigaciones sobre el orden de palabras en diferentes textos y registros del español y de otras lenguas.

Palabras clave: orden de palabras, estructuras gramaticales, tema/rema, Tópico/foco, organización del discurso, marcación.

\begin{abstract}
The present article analyzes the grammar phenomenon known as word order. Thus, an overview of different theoretical approaches and perspectives is proposed, which have attempted to systematize and explain the variations that speakers or writers make to the regular syntactic order with different purposes, conscious or not of the possibilities provided by their mother tongue grammar. The analysis focuses on three key aspects: a) theorizing about language b) conceptualization of the object in different positions/currents and c) its study in the Spanish language field. The suggested review makes it possible to understand the problem comprehensively within grammatical studies and lays the foundations for future research about word order in different texts and registers in Spanish and other languages.
\end{abstract}

Keywords: word order, grammatical structures, theme/rheme, Topic/focus, discourse organization, markation.

Recibido: 26 de diciembre de 2017

Aceptado: 11 de junio de 2018

\footnotetext{
${ }^{1}$ El presente trabajo de investigación fue realizado gracias a la Beca Doctorado Nacional CONICYT 2015, código: 21150119.
} 


\section{Introducción}

El estudio del orden de palabras ha sido un tema de reflexión desde tiempos remotos. Ha estado presente en el pensamiento lingüístico de la humanidad de diferentes maneras y a través de diversas aproximaciones, con los más variados objetivos científicos e investigativos: clasificar las lenguas del mundo, explicar fenómenos de la comunicación o describir el funcionamiento de las lenguas naturales, entre otros. La alteración de un orden regular, de un orden básico, de un orden neutro, ideal o "más común” en la cadena lingüística; ha llamado la atención de diferentes especialistas en el ámbito de los estudios gramaticales y lingüísticos. No obstante lo anterior, en general, la tradición gramatical hispánica no ha mostrado especial interés por el asunto, como ya lo advertía el profesor Rodolfo Lenz, iniciador de la lingüística chilena:

El orden de palabras en la oración castellana es, en general, muy libre y está gobernado más bien por el interés psicológico que por la estructura gramatical y lógica. La libertad de posición, de sujetos y complementos directos, ya antes, ya después del verbo, se debe, como vimos, a la posibilidad de distinguir la función del acusativo de la del nominativo, ya sea agregando la preposición al complemento, o ya expresando por medio del pronombre complementario, lógicamente superfluo, el caso substantivo. El orden de palabras en la oración castellana es un asunto todavía poco estudiado, la mayor parte de los gramáticos no dicen nada al respecto" (Lenz, 1920: 421)

El objetivo general de este artículo es, por lo tanto, analizar el concepto de orden de palabras en diferentes escuelas gramaticales. Para esto, se propone un recorrido por diferentes enfoques teóricos, tanto desde perspectivas formalistas como funcionalistas. Así, nuestros objetivos específicos son: a) analizar el tratamiento que se le ha dado al orden de palabras; b) determinar las implicancias del orden de palabras para la construcción de sentido; c) precisar la importancia del orden de palabras en la función comunicativa del lenguaje; d) identificar la distribución de la información en la oración como unidad plena; y e) distinguir las contribuciones que los distintos estudios sobre el orden de palabras han realizado a la teoría gramatical y a la descripción del español. 
A modo de proyección, se busca contar con los antecedentes teóricos y bibliográficos para desarrollar una descripción y análisis, tanto del orden de palabras en diferentes registros de la lengua española, como en su efecto en la organización de los textos.

En los apartados siguientes, se analiza el fenómeno del orden de palabras en el contexto del surgimiento de diferentes teorías lingüísticas; posteriormente, se propone un recorrido por diferentes enfoques teóricos, lo que permite realizar un análisis pormenorizado de cada una de las conceptualizaciones propuestas; finalmente el análisis se enfoca en el estudio del orden de palabras en español y en sus implicancias para la investigación de las funciones discursivas en diferentes tipos de textos.

\section{El orden de palabras y la teorización sobre el lenguaje}

2.1 Como indicamos en 1, el orden de palabras ha sido abordado por distintas tradiciones gramaticales desde los primeros tiempos. Algunas escuelas le han prestado escasa atención, pero han reconocido, sin embargo, un "orden natural de palabras"; este es el caso de los antiguos griegos:

Los griegos hablaban de un 'orden natural' de las palabras, un concepto que fue retomado a lo largo de la historia por diferentes escuelas gramaticales. Es el 'ordo naturalis' de las gramáticas medievales del tipo sujeto-verbo-objeto, postulando que falta claridad a toda oración que no empieza por su sujeto" (Montemayor-Borsinger, 2009: 19).

2.2 Los gramáticos de Port-Royal, en el siglo XVII, tampoco prestaron especial atención al orden, seguramente "llevados por las ideas de que el orden sintáctico refleja simplemente el orden natural del razonamiento y su estudio, por lo tanto, no es parte de la gramática" (Contreras, 1983: 34). Si bien Chomsky (1965) es un seguidor de la gramática de Port-Royal, no existe consenso en torno a esta idea al interior de la escuela transformacional. Este aspecto será desarrollado en el apartado 3.1.

2.3 Otro tanto ocurre con los seguidores del estructuralismo, para quienes la mayor parte de la sintaxis queda relegada al dominio del habla, ya que se trata de una variación individual que no es parte del sistema de unidades y relaciones que constituye la lengua como totalidad organizada. Lo que importa es la primacía de la lengua: 
Pero hay que reconocer que en el dominio del sintagma no hay límite señalado entre el hecho de lengua, testimonio del uso colectivo, y el hecho de habla, que depende de la libertad individual. En muchos casos es difícil clasificar una combinación de unidades, porque un factor y otro han concurrido para producirlo y en una proporción imposible de determinar (Saussure, 1916: 149).

2.3.1 Pese a lo anterior, el estructuralista norteamericano Joseph Greenberg (1963) aportó de manera considerable al estudio del orden de palabras. Estableció una tipología a partir del orden de los tres elementos universales del lenguaje: el sujeto, el verbo y el objeto. Mediante sus investigaciones e hipótesis tipológicas demostró que el encontrar propiedades universales en las lenguas no implica que todas ellas sean iguales en algún aspecto (Greenberg, 1963), por lo que estableció diferentes tipos y clasificaciones a partir de la combinación de estos tres constituyentes principales de la oración simple declarativa, que se constituyen en parámetros y correlaciones.

Así, mediante la comparación de un gran número de lenguas y la búsqueda en todas ellas de rasgos comunes o universales, el autor propone una clasificación de las lenguas de acuerdo con: a) su orden básico y b) parámetros adicionales referidos al uso de los casos, preposiciones y posposiciones, al orden relativo entre nombre y adjetivo y a la posición de los verbos auxiliares, entre otros. Su clasificación básica propone seis combinaciones posibles de sujeto (S), verbo (V) y objeto (O) y se aplica a distintos grupos de lenguas: a) SVO (español, inglés); b) SOV (alemán, latín, japonés); c) VSO (galés, árabe); d) VOS (malgache); e) OVS (parane); f) OSV (hixkaryana) (cf. Comrie, 1981: 41), siendo las dos primeras las más frecuentes y las dos últimas, prácticamente inexistentes. En este sentido, "puede considerarse al orden de las palabras como uno de los factores determinantes al momento de establecer una clasificación tipológica de las lenguas naturales" (Koza, 2011: 136).

2.3.2 La principal escuela estructuralista que teorizó sobre este problema fue el Círculo Lingüístico de Praga, pero antes de analizar su contribución se hace necesario revisar los postulados del francés de origen alemán Henri Weil (1844), considerado el iniciador del estudio de esta problemática para los estudiosos de la gramática y del texto.

Si bien los griegos, como se ha planteado, reconocían y postulaban el orden natural, eran conscientes de que este orden no siempre se respetaba o no se realizaba en la práctica. Es así que, motivado por esta observación de los filósofos antiguos, Weil (1844) se interesó por 
las violaciones a este orden natural: "en base a esto planteó la necesidad de buscar un principio rector para el orden de las palabras más allá de la sintaxis, independiente de las reglas de construcción gramatical llamadas en aquel entonces 'lógicas"” (MontemayorBorsinger, 2009: 19). Su argumento central es que las palabras son el signo o reflejo de las ideas, por lo que el orden de las palabras es un reflejo del orden de las ideas (Weil, 1844). En su tesis doctoral, propone una división de la oración en dos partes: a) noción inicial o punto de partida (point de départ) y b) propósito del discurso (but de discours):

“esta obra ha establecido fundamentos esenciales para la lingüística textual y funcional moderna. En particular su división de la oración será retomada un siglo después por la Escuela de Praga y la Lingüística sistémica funcional, en la forma de los conceptos de Tema y Rema" (Montemayor-Borsinger, 2009: 20).

Así las cosas, frente a la pregunta por el principio rector, desde el punto de vista del sentido común, que guía el orden de palabras, el estudioso francés postula que el encadenamiento sintáctico intenta proyectar la imagen del pensamiento y, en este sentido, el orden de palabras se desarrolla por analogía al proceso de pensamiento: para introducir lo que se quiere decir es necesario un punto de partida o de apoyo -situaciones existentes o conocidas-, para luego ir avanzando hacia elementos menos conocidos o desconocidos. Ahora bien, Weil (1844) reconoce que no siempre se sigue el orden noción inicial/propósito del discurso, que sería el orden mismo del pensamiento; también la secuencia puede ser invertida o el punto de partida puede ser eliminado: en estos casos habla de un orden patético ya que "el emisor se deja llevar por la emoción y no regula el hilo de su discurso, lo que redunda en la inversión de esos dos elementos" (Montemayor-Borsinger, 2009: 28).

De este modo, el orden de palabras es patético (Weil, 1844), emotivo (Mathesius, 1928 y Firbas, 1964) o marcado (Halliday, 1967) cuando la oración comienza con un complemento: "La tensión a nivel de gramática es mayor en el caso de complementos, ya que tiene una libertad de posición mucho menor que los circunstanciales, y a diferencia de estos, no pueden omitirse" (Di Tullio, 1997: 362). Se genera, así un orden de mayor impacto para el interlocutor en donde la mayor carga de emotividad se logra gracias a esta tensión gramatical. Todas estas reflexiones llevaron a Weil (1844) a proponer cambios en las formas de clasificar las lenguas por medio de las construcciones lingüísticas y, más precisamente, por medio de las estructuras oracionales: "Nuestras lenguas no siguen invariablemente un sistema exclusivo de 
construcción. El sujetar el orden de palabras a un sistema exclusivo sería una gran limitación, y de hecho Weil considera como mejores a las lenguas que imponen mínimas restricciones al orden de las palabras" (Montemayor-Borsinger, 2009: 37). Como se puede apreciar, este comparativista realizó también aportes a la tipología lingüística, aunque muchas de sus observaciones sobre las lenguas - como su clasificación de lenguas mejores o peores- no tienen sustento hoy, en el marco de la lingüística como disciplina científica (Firbas, 1974:11).

En síntesis, la visión de Henri Weil (1844) aportó con nociones que sentaron las bases para aproximaciones funcionalistas multipartitas: la división en dos de todo lo que decimos y la existencia de factores que van más allá de la dependencia gramatical para la explicación del flujo del discurso.

2.3.3 En el seno del estructuralismo, el Círculo Lingüístico de Praga es el movimiento intelectual que más atención brindó a este asunto. Fue su fundador, Mathesius (1928), el iniciador de la perspectiva funcional de la oración: "la oración se divide en dos partes, el tema, que contiene información ya conocida, y el rema, que presenta la información nueva" (Contreras, 1983: 34). Mathesius (1928), con su perspectiva funcional, retomó las ideas de Weil (1844), en especial, la que indica que el orden de palabras no está regido exclusivamente por principios de orden gramatical. En este sentido, el autor propone tres principios para el ordenamiento de las palabras: a) gramatical, centrado en la función sintáctica de las unidades; b) funcional, centrado en la distinción de tema y rema al interior de la oración y c) enfático, motivado por la necesidad de llamar la atención del receptor. Bajo estos principios, Mathesius (1928) distingue dos tipos de órdenes: a) objetivo, si el tema precede al rema; y b) subjetivo, si sucede lo contrario. Fernández Soriano (1993) utiliza también esta nomenclatura, cuando se altera la ordenación por medio de mecanismos de movimiento. En los siguientes ejemplos (Contreras, 1983:30) con entonación normal:

El dictador es un canalla

Este argumento no lo entiendo

Ambos tienen un orden objetivo. Ahora bien, si la cima melódica fuera el dictador y este argumento respectivamente, hablaríamos de un orden subjetivo, ya que éstos últimos sintagmas pasarían a constituir información remática (Contreras, 1983: 35). La importancia de la prosodia adquiere una relevancia tal, que es imposible desconocer los aspectos entonativos 
o de puntuación en el tratamiento de esta cuestión en las diferentes teorías: “en español como en muchas otras lenguas, la prominencia prosódica desempeña un papel fundamental en la identificación del foco" (Zubizarreta, 1999: 4228).

2.3.4 Los seguidores praguenses de Mathesius (1928), en especial Firbas (1964) y Daneš (1974), continuaron esta línea de trabajo. Así, Firbas (1964, 1966, 1974, 1986, 1992) analiza la perspectiva funcional de la oración con el objetivo de explicar cómo se distribuye la información en el acto de comunicar; para esto, introduce el concepto de dinamismo comunicativo, el que define como: "el grado en que el elemento de la oración contribuye al desarrollo de la comunicación, el grado en que este elemento, por así decirlo, impulsa la comunicación" (Firbas, 1966: 240). En este marco, se entiende que la estructura de la oración se orienta hacia un elemento que es el punto culmine de la comunicación intentada.

En esta línea, en su teoría temática, Firbas (1992) distingue los siguientes tipos de elementos: a) el tema (el elemento de menor dinamismo comunicativo), b) la transición que contempla los elementos que permite llegar hacia el núcleo del mensaje, c) el rema (el elemento que exhibe el más alto grado de dinamismo comunicativo). Existen tres factores que determinan la distribución de los diferentes grados de dinamismo comunicativo (DC): a) la modificación lineal, expresada en el orden en el que se desarrollan los elementos de la oración; b) el contextual, manifestado en que un elemento sea recuperable o no desde el texto precedente inmediatamente relevante; y c) el semántico, traducido en el tipo de contenido de los elementos y en el tipo de relaciones semánticas entre ellos.

El factor de modificación lineal permite un aumento gradual del DC, siempre y cuando ni el factor contextual ni el semántico compitan con él. El factor contextual es entendido por Firbas (1992) como el texto precedente, lo que puede recobrarse como contexto relevante. Respecto del factor semántico, el autor se enfoca en los tipos de verbos, puesto que lo que interesa es tanto el impacto del contenido semántico como las relaciones que se pueden establecer dependiendo de, por un lado, el modo en que los verbos introducen información nueva y, por otro, de si culminan o no el mensaje de las oraciones. En este último punto, será necesario analizar el objeto y su caracterización como elementos más o menos dinámicos en función de su papel en la culminación del propósito del mensaje. Este rol lo pueden cumplir: el objeto independiente del texto precedente relevante, el complemento del sujeto o del objeto independiente del texto precedente relevante, el grupo adverbial o preposicional del texto 
precedente relevante y un tipo de sujeto independiente del texto precedente relevante que viene después de verbos existenciales, como <haber> y <existir>.

Como decíamos, el factor contextual y el semántico pueden operar en contra del factor de modificación lineal, rompiendo lo que Firbas (1992) llama distribución básica del DC (tema-transición-rema), que da cuenta de cómo se manifiesta más plenamente: tema-rema, a través de un aumento gradual. Este orden "se realizaría en todas las lenguas indoeuropeas, pero con un grado de implementación variable de idioma a idioma. Por lo tanto, distribución básica no quiere decir necesariamente la más natural, la más frecuente o no marcada” (Montemayor-Borsinger, 2009: 49). En este sentido, Firbas (1986) destaca la naturaleza informacional del tema y del rema. El tema es el elemento con el grado más bajo de dinamismo comunicativo.

2.3.5 La teoría de la modificación lineal del estructuralista norteamericano Bolinger (1954) es coincidente con los postulados praguistas. Ambos comparten las observaciones sobre la organización de los elementos de la oración de izquierda a derecha en una ordenación de creciente especificidad. Veamos un ejemplo (Bolinger, 1954):

\section{¿Por qué, abruptamente, te retiraste?}

¿Por qué te retiraste abruptamente?

Para Bolinger (1954), el sentido de <abruptamente> en la primera oración es más amplio, menos específico que el de <te retiraste> y sucede lo contrario en el segundo ejemplo. En el segundo caso, el hablante puede tratar la oración entera como información nueva, en cambio, en la primera, solo el verbo puede considerarse como información nueva. Este y otros problemas, llevaron a los investigadores a cuestionar si realmente la modificación lineal explica esta asimetría entre estos dos tipos de oraciones, así como la existencia de reglas que asignen un estatuto particular a cláusulas como estas y a la jerarquización en la selección de los remas. Esto se puede relacionar con el debate posterior en Chomsky en relación con si las estructuras profundas tienen un orden lineal, según él defiende (1965) o si se trata de estructuras que se corresponden con sistemas de conjuntos no ordenados, como afirma Staal (1967), entre otros.

Para concluir con una primera aproximación a las ideas estructuralistas sobre este asunto, es relevante tener en cuenta que la noción de dinamismo comunicativo es una 
apreciación de cuánto contribuye un elemento de una cláusula al desarrollo de la comunicación. En ese sentido, al tratarse de una apreciación, es cuestionable poder afirmar qué tiene mayor o menor grado de DC, ya que sin un sustento empírico no es posible observar cuáles son los criterios que permiten calificar como mínima o máxima una contribución a la comunicación, que puede ser tanto información conocida como no conocida. En este contexto va a ser necesaria la incorporación de otros útiles conceptuales como la presuposición, la distinción entre estructura profunda y estructura superficial (orden profundo, orden superficial), marcación, foco, tópico entre otros elementos.

\section{El orden de palabras desde diferentes enfoques teóricos}

3.1 Al interior del paradigma generativista, existen diferentes posturas en relación con el orden de palabras. Chomsky (1965) considera, en primer lugar, que las estructuras profundas o subyacentes siguen un orden lineal, situación problemática respecto de la cual no existe consenso. En segundo lugar, encasilla el orden de palabras bajo el fenómeno de la actuación; en ese sentido, plantea que pueden existir reglas de actuación en aquellos hechos lingüísticos que no estarían regulados por la competencia, como por ejemplo el orden de palabras (Chomsky, 1965: 126-127). Lo gramatical sería, en este caso, optativo, facultativo, y, por lo tanto, se trataría de elementos opcionales, que caerían en el plano de lo estilístico. Dentro de lo opcional, estilístico, existirían normas o reglas de actuación; se trataría, por tanto, de meras reglas de reordenación estilística: "no son tanto reglas gramaticales como reglas de actuación" (Chomsky, 1965: 126). Esta clasificación del orden de palabras como un fenómeno perteneciente a la actuación se explica en parte por la escasa atención inicial que se le prestó a este mecanismo gramatical, tanto durante los primeros desarrollos del generativismo como al interior del estructuralismo norteamericano que vio crecer a Chomsky.

3.2 No obstante, la investigación al interior de la escuela transformacional ha realizado varios aportes y ha cuestionado su estatus como regla de reordenación estilística, ya que en estos casos se seguirían reglas bien definidas y no corresponderían a elecciones libres o dependientes de la mera voluntad del hablante: "El orden de las palabras es una de las marcas de función que licencia y manifiesta la organización de las estructuras sintácticas" (Di Tullio, 2010: 353). Esta concepción del orden como un recurso de marcación (Goded Rambau, 2000) es, a nuestro juicio, una de las propuestas más interesantes en este ámbito al interior de las 
teorías lingüísticas; en efecto, Goded Rambau (2000), desde una perspectiva funcionalcognitiva, considera que la marcación es una de las herramientas para el procesamiento y, en ese sentido, el orden de palabras se transforma en un recurso de marcación del que disponen las lenguas para hacer más prominentes ciertas unidades lingüísticas por sobre otras con diferentes finalidades.

3.3 En otra dimensión del problema, tanto la teoría estándar extendida (Chomsky, 1970) como la semántica generativa (Jackendoff, 1972) han relevado la importancia de los conceptos de información dada e información nueva, en el marco de los conceptos de presuposición y foco. Estas nociones que estructuran una nueva explicación generativista para la diferencia de oraciones señalan que se trataría más bien de un cuestión de gramática y no de estilo: "el foco es una frase que contiene la cima melódica; la presuposición, una expresión derivada por el reemplazo del foco por una variable" (Zubizarreta, 1999: 4222). Ambos conceptos serán claves para aproximarse al fenómeno desde perspectivas diversas.

3.4 Koktova (1999), a partir del principio de modificación lineal que se ha discutido hasta aquí y que fue introducido por la escuela de Praga, levanta una propuesta de teoría gramatical que toma como principio básico el orden de palabras; en su trabajo, pretende superar las limitaciones de las escuelas chomskyanas y praguistas, que conoce muy bien. Es así como, en su propuesta de gramática basada en el orden de palabras, busca determinar los recursos que provee la lengua natural para modificar la línea temporal de la oración, ya que su unidimensionalidad se considera una clara desventaja por el carácter irrecuperable de la información que transporta. En este sentido, para enfrentar esa linealidad, las lenguas han desarrollado recursos para intervenirla y hacer más efectiva la comunicación y los flujos de información. De esta manera:

"se concibe al orden de palabras como una estructura fascinante, altamente organizada, pulsante y segmentada, mediante la cual el lenguaje natural aprehende y modifica la linealidad temporal de la comunicación verbal y donde la (nueva) información de la cláusula se presenta de manera fragmentada" (Koza, 2011: 138).

Para explicar la fragmentación de esa línea temporal, la autora propone que las lenguas naturales utilizan ciertos mecanismos para modificar esta línea y propone a su vez cinco tipos de ordenamientos posibles: 
a) Orden de Palabras Profundo Fijo: tópico contrastivo-Tópico no contrastivo-foco.

Thus, I propose an (almost) obligatory leftward shift of Contrastive Topic as a communicatively important part of the sentence (second in importance only to the Focus) to the sentence-initial position (alternatively, it would be possible to postulate the order [CT-NT] as basic) (Koktova, 1999: 52).

b) Orden de Palabras Libre: libre distribución de tipos de relaciones sintácticas.

c) Orden de palabras superficial fijo: el orden fijo del inglés SVO-adv, por ejemplo.

d) Orden de palabras superficial libre que se corresponde con el orden de palabras profundo: incluye las topicalizaciones, como la dislocación a la izquierda, y la focalización, como la dislocación a la derecha.

e) Orden de palabras superficial libre que no se corresponde con el orden de palabras profundo:

"las dislocaciones a la izquierda de las scoping expressions hacia las posiciones de las scope ambiguas en inglés (y en otras lenguas) y el enfrentamiento enfático del foco (breve), usualmente hacia la posición inicial de la oración (y a veces, hacia la posición media de la oración)" (Koza, 2011: 142).

Si bien su noción de orden profundo es más bien operativa, ya que en su estudio lo considera como una noción útil, rescata uno de los elementos que han desatendido los transformacionalistas al clasificar el orden de palabras como una mera regla de reordenación estilística, ya que a nuestro juicio es importante que la partición esté considerada en el nivel profundo; así, los movimientos que se producen en el nivel de superficie toman posiciones que se corresponden con el orden profundo: los elementos del tópico tienden a situarse a la izquierda y los elementos del foco a la derecha.

Este hecho resalta la tensión que producen las alteraciones al orden regular, alteraciones que juegan un papel y que no constituyen, por tanto, un fenómeno secundario o sin importancia: "Las lenguas naturales deben compaginar la tendencia a la regularidad (la coincidencia entre las propuestas del Orden Profundo y el Orden de Superficie) con la tendencia de carácter psicolingüístico a la irregularidad (ruptura entre las dos estructuras), que tiene que ver con la necesidad de romper la línea temporal de la comunicación verbal” (Padilla García, 2001, 2005). 
3.5 Desde otra visión, Holan et al. (2000: 275) han elaborado una propuesta que les permite constituir una alternativa a la proyectividad. Según ellos, en el habla se pueden distinguir dos tipos de libertad de ordenamiento:

-weak word order freedom called freedom of constituent order within a continuous head domain where a constituent is free to move in several places but remains always continuous. The corresponding utterance respects therefore the constraint of projectivity. For instance:

(1) On the morning Paul used to go shopping.

-global word order freedom which corresponds on the opposite to a relaxation of continuity. In such cases, some extracted elements are allowed to appear out of the constituent they are supposed to belong to: the corresponding utterance is therefore non-projective.

(2) Who do you think that Mary claims that Sarah likes (Holan et al., 2000: 275).

Como podemos observar en la cita anterior, este enfoque teórico introduce el concepto de discontinuous constituency - relajación de la continuidad-, entendido como

La posibilidad de un constituyente de hacerse discontinuo, de intercalar ciertos elementos en su estructura básica, pero manteniendo su unidad interna. De acuerdo con esta concepción, el núcleo y los elementos que lo modifican se presentan como algo susceptible de intercalación, pero que a la vez mantiene su unidad estructural, lo que le va a permitir a Holan formalizar matemáticamente los posibles cambios del orden de palabras (Koza, 2011: 143).

Bajo el concepto de hole -hueco-, se indica una relación matemática entre el número de palabras de una oración y el número posible de huecos estructurales previos. Estos huecos estructurales pueden dar lugar a cambios en el ordenamiento, gracias a la intercalación de elementos que no pertenecen a ellos y que ocuparán el hueco, como sucede en el ejemplo (2) en donde se puede apreciar una extracción de wh- (Hudson, 2000).

Los dos tipos de órdenes presentados se analizan en base a la tarea de cheking grammar, consistente en verificar si una estructura pertenece o no a una determinada cadena estructural. Esta metodología permite obtener un resultado lineal o polinómico, es decir predecible, en donde los constituyentes discontinuos conforman un conjunto definido, por 
ejemplo, ciertos argumentos y palabras wh- (qu-) como se aprecia en el ejemplo (2). Lo que permite contar con un resultado predecible. Este trabajo de alta formalización y de vastas aplicaciones en lingüística computacional presenta problemas para su aplicación a las lenguas naturales, ya que el sistema de formalizaciones es muy complejo y, además, puede conducir a una definición circular, tal como plantea Padilla García (2001: 234) en su tesis doctoral sobre el orden en español coloquial. No obstante, resalta esta característica tan peculiar de las lenguas naturales de organizar de manera compleja las unidades informativas, lo que pone en evidencia que, junto con la linealidad, las lenguas verbales exhiben también una simultaneidad como propiedad o principio constitutivo (Simone, 1993).

3.6 En el terreno de teorías lingüísticas de corte funcionalista es necesario mencionar el aporte de Halliday (1967, 1994) y Halliday \& Mathiesen (2004) desde la Lingüística sistémico-funcional (LSF) en relación con el concepto de tema. La LSF define el lenguaje como sistema semiótico social que los hablantes emplean para construir significados en contexto, a través de opciones a diferentes niveles. Para Halliday (1994), las elecciones de significados están organizadas en tres componentes principales: a) la metafunción ideacional, relacionada con la organización del mundo que nos rodea, b) la metafunción interpersonal, asociada con el uso del lenguaje para el intercambio de significados con las personas con las que interactuamos y c) la metafunción textual, centrada en la organización de los significados ideativos e interpersonales dentro de textos coherentes y relevantes. De esta manera, las palabras realizan el significado de un texto y éste es concebido como una unidad semántica.

Halliday (1994) recoge de la Escuela de Praga los conceptos de tema y rema y define tema como la manifestación lexicogramatical de la metafunción textual que organiza significados ideacionales e interpersonales para formar textos: el tema es el punto de partida del mensaje. Este punto de partida debe incluir un elemento de la metafuncion ideacional: un participante, un proceso o un circunstancial, "para que realmente haya despegado el mensaje" (Montemayor-Borsinger, 2009: 95). El tema no marcado es para Halliday \& Matthiesen (2004), en el contexto de las declarativas en inglés, el sujeto gramatical, elemento obligatorio; en cambio, el tema marcado puede ser un objeto o un circunstancial: "The theme es the point of departure for the message. It is the element the speaker selects for grounding what he is going on to say" (Halliday \& Matthiesen, 2004: 58). La gramática sistémica funcional tiene la desventaja, a diferencia de otras propuestas, de estar enfocada exclusivamente en el inglés, 
por lo que sus desarrollos deben ser reflexionados y ajustados para su aplicación en otras lenguas. En el caso del español, el concepto de tema no marcado requiere su revisión y desarrollo para la lengua española. A continuación, analizaremos el estudio del orden de palabras en el ámbito lingüístico del español.

\section{El estudio del orden de palabras en español}

4.1 Los gramáticos del español no han centrado sus esfuerzos en la descripción del orden de palabras. Dentro de los gramáticos tradicionales, Gili Gaya (1961) es quien más se ocupó del asunto. Así, plantea: "El español participa de la tendencia general de las lenguas modernas hacia la construcción lineal o progresiva, en que el determinante sigue al determinado" (1961: 85).

4.2 En el campo del estructuralismo norteamericano, realizaron propuestas para el español Kahane \& Kahane (1950) y Bolinger (1954) a quien ya nos hemos referido.

4.3 En el ámbito del generativismo, una referencia obligada es Heles Contreras (1983) quien reafirma que el orden básico del español es SVO y analiza la posición de los distintos elementos en función de sus diferentes grados de rematicidad: la relevancia sintáctica y semántica del tema y rema.

4.4 Contreras (1983) a diferencia de los estudios anteriores, incorpora el componente prosódico o entonativo. Otro tanto realiza Fant (1984) con un estudio sintáctico y entonativo de las unidades informativas del español. El contexto en el que se encuentra la investigación sobre el orden de palabras lo resume muy claramente la Nueva Gramática de la Lengua Española:

El orden de palabras fue considerado tradicionalmente un recurso estilístico. Formaba parte de la llamada sintaxis figurada, es decir, del conjunto de recursos que permitía al hablante determinadas opciones expresivas que no alteraban las relaciones sintácticas básicas establecidas en la oración. Las alteraciones a las que se hace referencia no son vistas en la actualidad como recursos estilísticos, sino como diferencias entre posiciones sintácticas cuyo análisis constituye una parte fundamental del análisis gramatical. (RAE, 2009, pp. 2970-2971).

4.5 Zubizarreta (1999: 4217-4218) explica que las funciones informativas o discursivas de tema y foco son particularmente relevantes en la descripción de ciertos órdenes de palabras. Su investigación la lleva a dos grandes conclusiones generales: a) Algunas 
nociones de la gramática del discurso son relevantes a la hora de describir ciertos órdenes de palabras en el nivel de la cláusula; Estas nociones son las de ‘tema' (vs. Comentario) y 'foco' (vs. Presuposición). b) Para determinar si un cierto orden de palabras es adecuado en un discurso dado, deben tenerse en cuenta las propiedades entonativas asociadas a ese orden en particular, especialmente las asociadas al foco.

El tema, “aquello de lo cual trata la oración" (Zubizarreta, 1999: 4218), puede ser discursivo u oracional; en el primer caso solo proporciona información sobre el tema, es decir, “comenta sobre el tema" (Zubizarreta, 1999: 4218); en el segundo, corresponde al sujeto de un predicado lógico. El tema oracional, que es el que interesa a esta autora, se gramaticaliza a través de dos construcciones: la de tema vinculante y la de dislocación a la izquierda. El tema vinculante (hanging topic) introduce un cambio de tema discursivo y tiene las siguientes propiedades (Zubizarreta, 1999: 4224):

1. Aparece exclusivamente en la periferia izquierda de la cláusula matriz: El sillón, Pedro lo compró en el mercado de pulgas.

2. La relación entre el tema y una cierta posición dentro de la oración es una relación de correferencia; no existe relación de dependencia gramatical.

3. La relación no está restringida sintácticamente: el tema vinculante puede entrar en relación con cualquier posición dentro de la oración.

El sr. González, conocemos a la mujer que lo traicionó.

El sr. González, terminaremos la tarea antes de llamarlo.

El sr González, que María lo haya invitado sorprendió

a todo el mundo

(Zubizarreta, 1999).

En el caso de la dislocación a la izquierda, Zubizarreta (1999: 4224) distingue las siguientes propiedades:

1. El tema puede aparecer en la periferia izquierda de la cláusula matriz o en la periferia de la cláusula subordinada.

A sus amigos, María los invitó a cenar.

Estoy segura de que a sus amigos, María los invitó a cenar. 
2. Existe una dependencia gramatical entre el tema y la posición dentro de la cláusula con la cual se relaciona, como lo indica la presencia de la preposición adyacente al tema nominal en ciertos casos: Es seguro que de Juan, todo el mundo habla.

3. la relación está restringida sintácticamente: el tema no puede entrar en relación con una posición dentro de una cláusula relativa, de una clausula adverbial y de una cláusula de sujeto:

*Estoy segura de que a Pedro, conocemos a la mujer que lo traicionó.

Otro de los conceptos clave para entender la distribución de la información y las alteraciones al orden regular o canónico es la noción de foco, entendido a partir del concepto de presuposición discursiva de Chomsky (1970) y Jackendoff (1972) como "la parte no presupuesta de la oración" (Zubizarreta, 1999: 4224). El foco es parte del predicado, es decir, de la información que se predica del tema, ya que un mismo sintagma no puede funcionar como tema y foco al mismo tiempo. Esta propuesta se enfoca en un análisis, como hemos mencionado en el ítem 4.5, basado en la acentuación de la cláusula: "Los acentos tonales pueden ser altos, bajos, ascendentes o descendentes. Dentro del constituyente prosódico (o grupo melódico) una de las palabras se destaca como más prominente" (Zubizarreta, 1999: 4228). Esta prominencia prosódica cumple un papel relevante a la hora de identificar el foco, al punto de que en base al tipo de foco y al tipo de acento es posible describir diferentes órdenes de palabras con base sistemática: (i) verbo-objeto-sujeto, (ii) verbo-objeto-sintagma preposicional-sujeto y (iii) VPO.

4.6 Para Fernández Soriano (1993), el español, a diferencia de otras lenguas con estructura básica lineal, posee una flexibilidad mayor. Esta flexibilidad le permite modificar el orden sintáctico prototípico y hacer prevalecer otros factores de orden expresivo. En este contexto, la autora distingue dos tipos de órdenes: uno más fijo y otro más libre. Esta capacidad de la lengua española para alterar el orden básico está relacionada con la condición de visibilidad que establece que una:

Función semántica de 'agente'. 'paciente', 'destinatario', etc., de los distintos elementos nominales debe ser reconocible bien por medio de marcas formales (declinaciones, preposiciones, concordancia, etc.), bien por adyacencia estricta con el elemento que les asigna tal función (el verbo en este caso). (Fernández Soriano, 1993: 118) 
Así las cosas, es posible constituir explicaciones estrictamente gramaticales para las modificaciones del orden no marcado. Esto se debe al tipo de construcciones, a las propiedades del sujeto ${ }^{2}$, el tipo de verbo, la ergatividad, la inacusatividad, entre otros factores:

Así inciden sobre el orden de los constituyentes primordiales de la oración factores bien léxicos (la naturaleza del verbo), bien sintácticos (las diversas configuraciones), bien de estructura informativa (la distinción entre tema y rema), bien de procesamiento (la «pesantez» de determinadas cadenas). (Fernández Soriano, 1993: 148-149).

Esto evidencia que se trata de un fenómeno lingüístico complejo que debe seguir siendo investigado desde diferentes aproximaciones y teorías, ya que refleja en sus diferentes dimensiones la concatenación de elementos relativos a la generación, procesamiento y producción del lenguaje natural. Por ejemplo, la pesantez que refiere Fernández Soriano, es decir la tendencia de los objetos "pesados" a colocarse detrás, fue observada por Dik (1978) en el marco de su teoría sobre el orden preferido de los constituyentes independientes de las lenguas (LIPOC, por sus siglas en inglés):

"Y esto es válido para todos los constituyentes oracionales, no solo para el objeto: cuánto más largo y sobre todo más complejo estructuralmente sea un constituyente más posibilidades habrá de que vaya situado, en cualquier lengua considerada, al final de la oración” (Padilla García X., 2000: 226).

Esto resulta muy interesante, a nuestro juicio, ya que tiene implicancias cognitivas, de procesamiento, y, por lo tanto, de la construcción de las representaciones mentales de los hablantes/escritores.

4.7 Desde la teoría de principios y parámetros existen diferentes explicaciones e hipótesis para esta problemática; Zagona (2002), por ejemplo, realiza algunas propuestas para las cláusulas declarativas. La flexibilidad del orden del sujeto en español (FLEX), la posibilidad de que este pueda aparecer en diferentes posiciones y la supuesta optatividad de los sujetos preverbales configuran un conjunto de problemas que desde el generativismo se ha tratado de explicar y, como hemos visto, redundan en el hecho de que "el orden de los constituyentes es sensible a su contenido informativo" (Zagona, 2002: 226). La pregunta

\footnotetext{
${ }^{2}$ Existen sujetos obligatoriamente postverbales, en el caso de estructuras interrogativas, u obligatoriamente preverbales, generalmente los sintagmas nominales con determinante: "la optatividad de los sujetos preverbales no es fácil de explicar” (Zagona, 2002: 224).
} 
básica es ¿qué propiedades del español hacen posible este orden variable? "En español los rasgos verbales de FLEX son fuertes, mientras que en inglés son débiles.” (Zagona, 2002: 229).

Una primera posibilidad para explicar el cambio de orden dice relación con las funciones del discurso foco y tópico, dos conceptos que son centrales para la estructura informativa. El sujeto y el verbo contienen información "dada" o "conocida" en virtud del contexto. Comrie (1981) analiza la información nueva como foco y los demás constituyentes como no foco; la oración puede incluir, entonces, los siguientes rasgos: +foco (información nueva) o -foco (información presupuesta).

Zubizarreta (1999), como mencionábamos en 4.6, propone que esta división deriva de la noción de presuposición. Lo que el hablante y el oyente suponen como verdadero en el momento de la enunciación es "presupuesto"; los elementos no presupuestos constituyen el foco de la oración (Zagona, 2002: 233). En este sentido, el tópico corresponde a lo que el hablante y el oyente presuponen: "los tópicos se caracterizan por aparecer en posiciones periféricas, separadas del "comentario" por la entonación” (Zagona, 2002: 237). Ya que como han planteado diferentes autores existe una relación estrecha entre foco, entonación y orden de palabras -asociación sistemática-, el elemento tópico cumpliría una función:

$\mathrm{Si}$ suponemos que en algunas lenguas el rasgo [TÓPICO] se gramaticaliza como categoría funcional, se predice que estas lenguas permitirán que cualquier elemento portador de este rasgo se desplace al especificador de SF. La existencia de oraciones con el orden SX-VSO en español es una prueba a favor de esta hipótesis. La variedad de constituyentes que pueden ser tópicos nos permite dar cuenta de las diferentes posiciones en que puede aparecer el sujeto (Zagona, 2002: 241).

El tópico como rasgo funcional distinto de FLEX permite el desplazamiento de constituyentes; así, el rasgo tópico puede desplazar y atraer al sujeto o a otros constituyentes. Esta categoría funcional se relaciona entonces con el papel que cumplirían los constituyentes en el discurso. Así Sintagma Flexión puede funcionar como sintagma Tópico, lo que permite que los tópicos se desplacen a su especificador (Zagona, 2002: 238). Aunque a través de este rasgo funcional es posible captar algunas propiedades del orden en las oraciones declarativas, siempre en términos de desplazamiento, para cotejar rasgos, con tópicos únicos y que se 
comporten como elementos focales, es importante considerar que las oraciones pueden tener más de un tópico.

4.8 Finalmente, otra explicación se relaciona con el parámetro del sujeto nulo. Este establece una relación entre la libertad de orden del sujeto y la posibilidad de sujetos tácitos o nulos. En efecto, estos estudios comparten los siguientes principios:

Propiedades de la flexión rica:

a. Una flexión de rasgos de concordancia rica permite la identificación de los pronombres nulos.

b. una flexión de concordancia rica afecta la rección del sujeto, lo que a su vez afecta la asignación de caso (Zagona, 2002: 256).

En este sentido, lenguas como el español y el italiano admiten sujetos nulos y presentan una rica flexión, a diferencia de, por ejemplo, el inglés y el francés, que no admiten sujetos nulos y poseen una flexión verbal mínima. Los parámetros sintácticos se derivan entonces a partir del contraste entre rasgos fuertes y rasgos débiles:

En inglés los rasgos nominales fuertes de FLEX pueden ser cotejados solamente por ascenso del sujeto al especificador de SF. En español, en cambio, los rasgos de concordancia del verbo cotejan los rasgos nominales de FLEX y no se requiere el desplazamiento del sujeto" (Zagona, 2002: 263).

4.9 Según nuestro parecer, todas estas propuestas deben seguir siendo exploradas para así entender de mejor manera el proceso de expansión discursiva, de incrementación y de modificación de la información compartida, fenómenos todos que indudablemente tienen un sustrato gramatical poderoso que actúa en el manejo de los flujos de información para la construcción del sentido.

\section{Reflexiones finales}

En el presente artículo, se han revisado los principales aportes que diferentes escuelas y enfoques han realizado al estudio del orden de palabras en las lenguas naturales. Se trata de un asunto en el que los estudios gramaticales han tenido una actitud vacilante: en general, han despreciado o desatendido su estudio; como contrapartida, se han levantado propuestas que rescatan su importancia al interior de diversas escuelas lingüísticas. La Figura 1 muestra una síntesis comparativa de las principales conceptualizaciones que ha recibido el fenómeno. 
Artículo. Enrique Sologuren Insúa, María Natalia Castillo Fadić. El orden de palabras en los estudios gramaticales: un recorrido a través de diferentes perspectivas lingüísticas

\section{Figura 1: síntesis comparativa de las formas de abordar el orden de palabras}

\begin{tabular}{|c|c|c|}
\hline Escuela & Autores & Conceptualización del orden de palabras \\
\hline $\begin{array}{l}\text { Neogramática } \\
\text { Comparativismo }\end{array}$ & Weill (1844) & $\begin{array}{l}\text { Reflejo del pensamiento. } \\
\text { Ordo naturalis: presente en las gramáticas antiguas y medievales. }\end{array}$ \\
\hline $\begin{array}{l}\text { Círculo lingüístico de } \\
\text { Praga }\end{array}$ & $\begin{array}{l}\text { Mathesius } \\
(1928) \\
\text { Firbas (1964) }\end{array}$ & $\begin{array}{l}\text { Perspectiva funcional de la oración. } \\
\text { Tema-rema desarrollo del mensaje a partir de su dinamismo comunicativo. }\end{array}$ \\
\hline $\begin{array}{l}\text { Estructuralismo } \\
\text { norteamericano }\end{array}$ & $\begin{array}{l}\text { Bolinger } \\
(1954)\end{array}$ & Teoría de la modificación lineal. \\
\hline $\begin{array}{l}\text { Estructuralismo } \\
\text { norteamericano } \\
\text { Tipología } \\
\text { Comparativismo de } \\
\text { nuevo cuño. }\end{array}$ & $\begin{array}{l}\text { Greenberg } \\
(1963)\end{array}$ & $\begin{array}{l}\text { Factor determinante a la hora de establecer una clasificación de las lenguas } \\
\text { del mundo. }\end{array}$ \\
\hline $\begin{array}{l}\text { Generativismo } \\
\text { Aspectos de una teoría } \\
\text { de la sintaxis }\end{array}$ & $\begin{array}{l}\text { Chomsky } \\
(1965)\end{array}$ & $\begin{array}{l}\text { Regla de reordenación estilística } \\
\text { Rule of performance }\end{array}$ \\
\hline $\begin{array}{l}\text { Generativismo } \\
\text { Teoría estándar } \\
\text { extendida y semántica } \\
\text { generativa }\end{array}$ & $\begin{array}{l}\text { Chomsky } \\
(1970) \\
\text { Jackendoff } \\
(1972) \\
\text { Contreras } \\
(1983)\end{array}$ & $\begin{array}{l}\text { Presuposición y foco en la estructura superficial. } \\
\text { Presuposición y foco como parte de la estructura semántica de la oración. } \\
\text { La jerarquía remática está determinada por la estructura semántica de la } \\
\text { oración. }\end{array}$ \\
\hline Gramática funcional & Dik (1978) & LIPOC \\
\hline $\begin{array}{l}\text { Gramática basada en el } \\
\text { orden de palabras }\end{array}$ & $\begin{array}{l}\text { Koktova } \\
(1999)\end{array}$ & Segmentación y pulsación de la oración. Fragmentación. \\
\hline $\begin{array}{l}\text { Lingüística } \\
\text { computacional }\end{array}$ & $\begin{array}{l}\text { Holan et al } \\
(2000)\end{array}$ & Constituyente discontinuo, intercalación y hueco estructural. \\
\hline $\begin{array}{l}\text { Lingüística } \text { sistémico } \\
\text { funcional }\end{array}$ & $\begin{array}{l}\text { Halliday } \\
(1994) \\
\text { Halliday \& } \\
\text { Matthiesen } \\
\text { 2004) }\end{array}$ & $\begin{array}{l}\text { Tema-rema } \\
\text { Tema marcado y no marcado metafunción textual. } \\
\text { Primer elemento experiencial }\end{array}$ \\
\hline $\begin{array}{l}\text { Generativismo } \\
\text { Principios } \quad y \\
\text { parámetros }\end{array}$ & $\begin{array}{l}\text { Chomsky } \\
(1981) \\
\text { Zagona } \\
(2002)\end{array}$ & $\begin{array}{l}\text { Funciones discursivas: tópico y foco. } \\
\text { Contraste entre rasgos fuertes y débiles. } \\
\text { Parámetro del sujeto nulo }\end{array}$ \\
\hline Perspectiva cognitiva & $\begin{array}{l}\text { Goded } \\
\text { Rambau } \\
(2000)\end{array}$ & $\begin{array}{l}\text { Teoría de la marcación. } \\
\text { Marca de procesamiento. }\end{array}$ \\
\hline
\end{tabular}


La Figura 1 sintetiza en una tabla, que no pretende ser exhaustiva, la gran diversidad de corrientes, enfoques y útiles conceptuales que se utilizan y se ha utilizado para la descripción y explicación de este fenómeno.

Esperamos que esta revisión constituya un aporte a la comprensión de la naturaleza y el alcance del orden de palabras en el marco de la teorización sobre el lenguaje y su impacto en la descripción de la gramática del español; asimismo, confiamos en que contribuya a reconocer los factores gramaticales que entran en juego, pero también los textuales, cognitivos, comunicativos y tipológicos en un proceso de interrelación entre forma y función comunicativa, como plantea Padilla García (2000: 222):

Las palabras se ordenan en el discurso en virtud de estos dos principios o componentes (sintáctico y pragmático) y ambos forman parte de un continuum, cuya función principal es reconciliar la necesidad de ser imperiosamente comunicativo (orden pragmático) con la obligación de ser claro y ordenado (orden sintáctico).

Estos dos principios se encuentran también presentes en las ideas de Dik (1978) Givón (1979) y Payne (1992) desde perspectivas funcionalistas y cognitivas. La tensión a nivel de gramática y a nivel de la pragmática (Padilla García, 2005) son elementos que deben seguir siendo explorados.

Ahora bien, desde una perspectiva metodológica, consideramos que esta revisión debe ser complementada con un análisis de corpus en diferentes tipos de textos y en diferentes registros, ya que la variación lingüística es una propiedad intrínseca de las lenguas naturales: los recursos lingüísticos preferenciales, así como los órdenes preferidos que seleccionen determinados tipos de textos van a influir en las posiciones sintácticas. En esta línea, resulta de interés desarrollar investigaciones que relacionen el orden de palabras con diferentes géneros del discurso, ya que "La unidad de significado que es el enunciado textual siempre está en función del plan temático del escritor. En ese sentido, cualquier modificación de la estructura informativa del texto repercute, de modo directo, en el contenido y distribución de los enunciados textuales" (Figueras, 2001: 63).

Al analizar la diversidad de conceptualizaciones y formas de abordar el orden de palabras, podemos notar que en la base de todas se encuentra el problema de cómo organizar, distribuir, expandir, incrementar o modificar la información que compartimos, a través de los mecanismos gramaticales de la lengua. Para resolver dicho problema, serán necesarios 
estudios experimentales sobre el procesamiento psicolingüístico de diferentes formas de explotar los ordenamientos sintácticos posibles y probables en diferentes lenguas.

Finalmente, esta revisión proporciona una primera aproximación para proponer investigaciones en el ámbito de la gramática del discurso, ámbito aún poco explorado en el ámbito del español y en el establecimiento de gramáticas locales asociadas a diferentes géneros, registros y tipos textuales especializados y no especializados, para así comprender mejor cómo los hablantes usan el lenguaje, cómo usan su conocimiento gramatical y lo moldean en función de una diversidad de objetivos que la interacción y la vida humana en sociedad demandan. Debemos, por tanto, seguir reflexionando sobre el lenguaje y sobre esta central eléctrica, como la llaman Halliday \& Matthiessen (2004), de los procesos de comprensión y construcción del conocimiento: la gramática.

\section{Bibliografía}

Bolinger, Dwight. "English Prosodic Stress and Spanish Sentence Order", Hispania (1954):152-156.

Chomsky, Noam. Aspects of the Theory of Sintax . Cambridge, Massachusetts, USA: The M.LT. Press Massachusetts Institute of Technology Cambridge, Massachusetts 1965.

Chomsky, Noam. Some Empirical Issues in the Theory of Transformational Grammar. Indiana, USA: University Club, 1970.

Chomsky, Noam. Lectures on Government and Binding.Dordrecht, Netherlands: Claredon Press, 1981.

Comrie, Bernard. Universales del lenguaje y tipología lingüística. Madrid: Gredos, 1981.

Contreras, Heles. El orden de palabras en español.Madrid: Cátedra, 1983.

Daneš, František. "Functional Sentence Perspective and the Organization of the Text". Papers on Functional Sentence Perspective. Praga, República Checa: Mouton Academic Publishing House, 1974. 11-37.

Di Tullio, Ángela. Manual de gramática del español. Buenos Aires,Argentina: Edicial, 1997.

Di Tullio, Ángela. Manual de gramática del español. Buenos Aires, Argentina: Waldhuter, 2010.

Dik, Simon. Gramática funcional. Madrid: SGEL, 1978.

Fant, Lars. Estructura informativa en español: estudio sintáctico y entonativo. Tesis doctoral. Uppsala, Suecia: Uppsala University, 1984.

Fernández Soriano, Olga. "Sobre el orden de palabras en español", Dicenda. Cuadernos de filología hispánica 11 (1993): 113-152.

Figueras, Carolina. Pragmática de la puntuación.Barcelona, España: Octaedro, 2001.

Firbas, Jan. "On defining the Theme in Functional sentence Analysis", TLP, Travaux Linguistiques Pragues 1(1964): 267-280.

Firbas, Jan. "Non Thematic Subjects in Contemporary English", TLP, Travaux Linguistique Prague 2 (1966): 239-256. 
Firbas, Jan. "Some Aspects of the Czechoslovak Approach to Problems of Functional Sentence Perspective", ed. Danes, Frantisek. Papers on Functional Sentence Perspective. Praga, República Checa: Mouton Academic Publishing House, 1974. 1137.

Firbas, Jan. "On the Dynamics of Written Comunication in the Ligth of the Theory of Functional Sentence Perspective". Studying Writing: Linguistics Approaches, orgs. Charles Cooper, y Sidney Greenbaum. Beverly Hills, USA: Sage, 1986. 40-71.

Firbas, Jan. Functional Sentence Perspective in Written and Spoken Communication. Cambridge, USA: University Press, 1992.

Gili Gaya, Samuel. Curso superior de sintaxis española.Barcelona, España: VOX, 1961.

Givon, Talmy. "From Discourse to Sintax: Grammar as a Processing Strategy". Sintax and Semantics: Discourse and Sintax. NY: Academic Press, 1979. 81-112.

Goded Rambau, Margarita. Orden de palabra y categorización lingüística. Madrid: Universidad Autónoma de Madrid, 2000.

Greenberg, Joseph. Universal of Language.Cambridge, USA: MIT press, 1963.

Halliday, Michael. "Notes on Transitivity and Theme in English", Journal of Linguistics 1 (1967): 31-81.

Halliday, Michael. An Introduction to Functional Grammar. London: Arnold, 1994.

Halliday, Michael y Matthiessen, Christian. An Introduction to Functional Grammar (3 Ed.). London: Edward Arnold, 2004.

Holan, Tomas, Kubon, Vladislav, Oliva, Karel y Plátek, Martin. "On Complexity of Word Order", TAL, Travaux Linguistique Prague 41.1 (2000): 273-300.

Hudson, Richard. "Discontinuity". TAL, Traitement Automatique des Langues 41.1(2000): 15-56.

Jackendoff, Ray. Semantic Interpretation in Generative Grammar. Cambridge, USA: MIT press, 1972.

Kahane, Henry y Kahane, Renée. "The Position of the Actor Expression in Colloquial Mexican Spanish", Language 26 (1950): 236-263.

Koktova, Eva. Word-order Based Grammar. Berlín: Mouton de Gruyter,1999.

Koza, Walter. Los signos de puntuación en el análisis automático de textos. El caso de la coma. Tesis Doctoral. Rosario, Argentina: Universidad Nacional de Rosario, 2011.

Lenz, Rodolfo. La oración y sus partes. Santiago, Chile: Nascimento, 1920.

Mathesius, Vilém. "On Linguistic Characterology with Ilustrations from Modern English". Ed. Joseph Vachek A Prague School Reader in Linguistics. Reprinted. Indiana, USA: University Press, (1928(1964)). 59-67.

Montemayor-Borsinger, Ann. Tema. Una perspectiva funcional de la organización del discurso. Buenos Aires: Eudeba, 2009.

Padilla García, Xose. "El orden de palabras". Ed. Antonio Briz. ¿Cómo se comenta un texto coloquial? Barcelona, España: Ariel, 2000. 221-241.

Padilla García, Xose. El orden de palabras en el español coloquial. Tesis doctoral. Valencia, España: Universidad de Valencia, 2001.

Payne, Doris. Pragmatics of Word Order Flexibility . Amsterdam, Netherlands: J. Benjamins, 1992.

RAE. Nueva gramática de la lengua española. Madrid: Espasa, 2009.

Saussure, Ferdinand de. Curso de Lingüística General. Buenos Aires, Argentina: Losada, 1916. 
Simone, Raffaele. Fundamentos de lingüística.Barcelona, España: Ariel, 1993.

Staal, Johan Frederik. Word Order in Sankrist and Universal Grammar. Foundations of Language Supplementary Series 5. Dordrecht, Netherlands: D. Reidel Publishing Company, 1967.

Weil, Henri. De l'ordre des mots dans les langues anciennes comparées aux langues modernes.Paris: Libraire Franck, 1844.

Zagona, Karen. Sintaxis generativa del español. Madrid: Visor Libros, 2002.

Zubizarreta, María Luisa. "Las funciones informativas: tema y foco". Gramática descriptiva de la lengua española,orgs. Ed. Violeta Demonte y Ignacio Bosque. Madrid: Espasa, 1999. 4216-4244. 